\section{Persistent hiccups as the presenting symptom of a pulmonary embolism}

\author{
Steve J. Durning, ${ }^{1}$ David J. Shaw, ${ }^{1}$ \\ Anthony J. Oliva, ${ }^{2}$ Michael J. Morris ${ }^{1}$ \\ ${ }^{1}$ Internal Medicine Residency; ${ }^{2}$ Internal \\ Medicine Clinic, Department of Medicine, \\ Brooke Army Medical Center, \\ Fort Sam Houston, TX, USA
}

\section{Abstract}

An 81-year-old male with a history of symptomatic bradycardia controlled by a pacemaker presented to our institution with three days of persistent hiccups. He denied any pulmonary symptoms and his initial evaluation showed no evidence of tachypnea, tachycardia, or hypoxia. Pacemaker malfunction or migration of the pacer leads was ruled out as an etiology and no intracranial pathology was present. Admission chest radiograph was normal but a computed tomography of the chest demonstrated a left lower lobe pulmonary embolus. After treatment with anticoagulation was initiated, the hiccups resolved within the next week. This is a unique case presentation of hiccups as the only presenting symptom of an otherwise asymptomatic pulmonary embolism.

\section{Introduction}

Hiccups (referred to as singultus in the medical literature) are a common occurrence and rarely have a pathological cause, but can be indicative of an underlying medical condition. Most episodes of hiccups are transient and resolve spontaneously, but if they last longer than $48 \mathrm{~h}$ they are termed persistent hiccups, and if persist beyond 30 days, they are considered intractable. Transient hiccups are usually benign, occur in most individuals spontaneously but can be triggered by numerous causes to include overeating, carbonated beverages, aerophagia, gastric insufflation during endoscopy, gastroesophageal reflux, sudden changes in ambient or gastrointestinal temperature, alcohol use, sudden excitement, or emotional stress. ${ }^{1}$ Transient hiccups may be irritating at most, but when they are persistent beyond $48 \mathrm{~h}$, they often portend an organic cause such as intracranial or intrathoracic pathology. ${ }^{2}$ There have been several reported cases of other intrapulmonary causes of hiccups (masses, pneumonia, etc.), but this is the first reported case of a documented pulmonary embolus presenting with the sole symptom of persistent hiccups.

\section{Case Report}

An 81-year-old male presented to our institution with the primary complaint of hiccups for the previous three days. He characterized the hiccups as constant with only intermittent breaks, continuous through his sleep, and not associated with any new activity or dietary habits that precipitated the hiccups or caused them to break. Upon presentation in the emergency department, he was otherwise asymptomatic and denied any abdominal pain, chest pain, dyspnea, or neurological symptoms. His only medications were acetaminophen and as needed meloxicam for knee osteoarthritis. Significant past medical history included symptomatic bradycardia resulting in syncope and placement of a dual chamber pacemaker five years ago. His pacemaker had been functioning properly and was last interrogated two years prior to admission.

On exam, his blood pressure was 147/68, heart rate of 63 , and respiratory rate at 20 times per minute with intermittent hiccups and oxygen saturation of $98 \%$ on room air. Physical exam revealed a thin male in no distress who was hiccupping throughout the exam. Closer examination of Head, Eyes, Ears, Nose and Throat, pulmonary, cardiovascular and neurologic systems was unremarkable. A portable chest radiograph was negative for acute cardiopulmonary pathology, and demonstrated the unchanged appearance of the 2lead cardiac pacer within the left chest compared to prior chest radiograph done one month earlier. Evaluation for acute coronary syndrome was negative with an unchanged electrocardiogram from baseline and three negative sets of cardiac enzymes. To evaluate for additional pathologic causes of hiccups, computed tomography (CT) of the head with contrast was performed and showed no evidence of intracranial pathology. After the addition of thorazine $25 \mathrm{mg}$ three times daily, the patient experienced transient relief for an hour, but the hiccups again returned. To further evaluate other uncommon causes of persistent hiccups, a contrast-enhanced CT of the chest and abdomen was performed to rule out a thoracic mass causing irritation of the diaphragm or phrenic nerve. A significant filling defect was seen involving the anterior and lateral segments of the left lower lobe pulmonary artery (Figure 1). The pacemaker leads were properly positioned and no pleural effusion or frank infarction of the lung was identified.

The patient was begun on systemic anticoagulation with subcutaneous enoxaparin and oral
Correspondence: Steven J. Durning, Brooke Army Medical Center, 3851 Roger Brooke Drive, Fort Sam Houston, TX 78234, USA.

E-mail: steve.durning55@gmail.com

Key words: hiccups, pulmonary embolism.

Conflicts of interests: the authors report no potential conflicts of interests. The opinions or assertions contained herein are the private views of the authors and are not to be construed as the reflecting the Department of the Army, Department of the Air Force, or the Department of Defense.

Received for publication: 13 July 2011.

Revision received: 8 January 2012.

Accepted for publication: 12 January 2012.

This work is licensed under a Creative Commons Attribution NonCommercial 3.0 License (CC BYNC 3.0).

@C Copyright S.J. Durning et al., 2012

Licensee PAGEPress, Italy

Chest Disease Reports 2012; 2:e2

doi:10.4081/cdr.2012.e2

warfarin for pulmonary embolism. Further evaluation for deep venous thrombosis with lower extremity duplex ultrasonagraphy was negative and no underlying malignancy was identified. The patient denied any risk factors for venous thromboembolism to include travel, prolonged immobilization, injury, or prior history of pulmonary embolism. He was discharged on thorazine and benzonatate for symptomatic relief of his hiccups. At his follow-up evaluation, the patient's hiccups had resolved within two weeks on chronic anticoagulation.

\section{Discussion}

Persistent hiccups as the presenting symptom for an otherwise asymptomatic pulmonary embolism has rarely been described in the medical literature. In this unusual presentation, the patient had no typical pulmonary embolism symptoms such as dyspnea or pleuritic chest pain, which have been reported in $44 \%$ to $74 \%$ of patients respectively. ${ }^{3}$ Hiccups that persist for greater than $48 \mathrm{~h}$ should prompt the evaluation for a pathologic cause and clinicians need to be wary not to dismiss persistent hiccups when a cause with significant morbidity could have remained undiagnosed.

A hiccup is defined as a sudden diaphragmatic spasm, which causes a sudden inhalation which is abruptly interrupted by the closure of the glottis. The characteristic sound produced by the spasmodic closing of the glottis is what has led to the term hiccup. ${ }^{2,4}$ There 
are numerous potential causes of persistent hiccups, and they may be grouped under the following categories: central nervous system, head and neck, thoracic, gastrointestinal, metabolic, postoperative, psychogenic, and idiopathic. Commonly reported causes may include ischemic/hemorrhagic stroke, encephalitis, head trauma, goiter, pharyngitis, intrathoracic lymphadenopathy, and alcohol intoxication. Central nervous system causes of hiccups may include a wide variety of vascular, infectious, and structural disorders. A wide variety of gastrointestinal disorders has also been described ranging involving esophageal (reflux esophagitis and distention), gastric (gastritis, peptic ulcer disease, distention, carcinoma) and pancreatic (pancreatitis, cancer) causes. In a review of hospital admissions for hiccups, Cymet identified 54 patients with a diagnosis of hiccups, $22 \%$ with a primary diagnosis and $78 \%$ with a secondary diagnosis. All of the patients who had hiccups for $48 \mathrm{~h}$ or more (52\%) had an underlying disease or pathology that caused the hiccups. ${ }^{5}$ Although this report did not specify the exact etiologies of hiccups, the gastroenterology service was

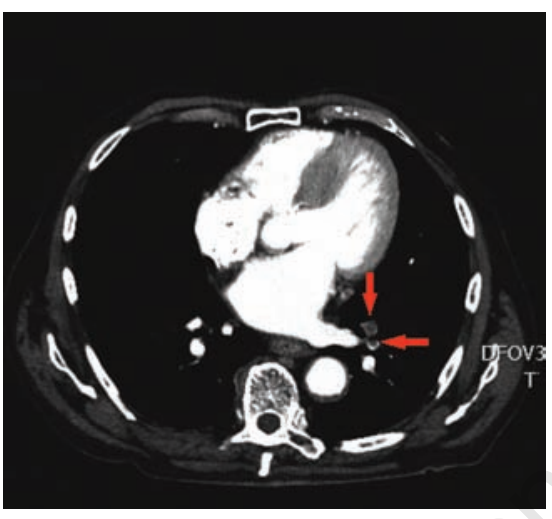

Figure 1. Computed tomography image of the chest, demonstrating evidence of clot in the anterior and lateral segments of the left lower lobe pulmonary artery (red arrows). the most frequent consulted ( $13 \%$ of patients) and esophagogastroduodenoscopy was normal in five patients.

While thoracic causes of hiccups include esophageal, mediastinal, and diaphragmatic disorders, intrapulmonary pathology has been rarely reported as the cause of intractable hiccups and includes lung masses and pneumonia. ${ }^{6,7}$ Other reported pulmonary causes may include bronchitis, empyema, pleuritis, asthma, or chest trauma. There is one case of a pulmonary embolus reported as the cause of intractable hiccups. ${ }^{8}$ However, the patient had an untreatable non-small cell lung cancer and the diagnosis of pulmonary embolism was never established. A thrombus was found in the inferior vena cava but no imaging of the chest was ever performed.

In our presented case, the only abnormality after extensive evaluation was the presence of a left lower lobe pulmonary embolus with the absence of any mass or other factor that could initiate the hiccup reflex arc. The location of the pulmonary embolus (anterior and lateral lower lobe segments of pulmonary artery) may have provided the proper nidus for irritation of the phrenic nerve and the subsequent development of hiccups. The physiology of hiccups is not entirely understood, but involves a reflex arc which involves an afferent loop mediated by the phrenic and vagus nerves as well as from the sympathetic chain of T-6 to T-12.,9,10 The phrenic nerve which innervates the diaphragm mediates the efferent loop, but there has also been suggestion that the accessory muscles of inspiration and the glottis may mediate part of the efferent loop.

After extensive evaluation for hiccups that were previously unremitting, the only pathologic finding was a pulmonary embolism. The subsequent improvement of hiccups upon starting anticoagulation would argue in fact that the pulmonary embolism was the underlying cause. The pulmonary embolism identified in this case was not small as it completely occluded the anterior and lateral primary branches of the left lower lobe pulmonary artery. It is theoretically possible that the hic- cups were idiopathic and it was coincidental that the patient had a pulmonary embolism while the hiccups resolved concurrently with anticoagulation; however this seems more unlikely than the proposed mechanism in this case. This case demonstrates the importance of a thorough evaluation for underlying causes in persistent hiccups to include central nervous system and thoracic imaging, which may identify the underlying cause.

\section{References}

1. Schuchmann JA, Browne BA. Persistent hiccups during rehabilitation hospitalization: three case reports and review of the literature. Am J Phys Med Rehabil 2007; 86:1013-8.

2. Souadjian JV, Cain JC. Intractable hiccup. Etiologic factors in 220 cases. Postgrad Med 1968;43:72-7.

3. Prendergast TJ, Ruoss SJ, Seeley EJ. Chapter 9: Pulmonary disease. In: McPhee SM, Hammer GD. Pathophysiology of disease: an introduction to clinical medicine, 6th ed. New York, NY: McGraw-Hill; 2010.

4. Kolodzik PW, Eilers MA. Hiccups (singultus): review and approach to management. Ann Emerg Med 1991;20:565-73.

5. Cymet TC. Retrospective analysis of hiccups in patients at a community hospital from 1995-2000. J Natl Med Assoc 2002;94: 480-3.

6. Yamazaki Y, Sugiura T, Kurokawa K. Sinister hiccups. Lancet 2008;371:1550.

7. Burdette SD, Marinella MA. Pneumonia presenting as singultus. Southern Med J 2004;97:915.

8. Zylicz Z. Intractable hiccups caused by pulmonary embolism. A case report. Adv Pall Med 2010;9:149-52.

9. Lewis JH. Hiccups: causes and cures. J Clin Gastroenterol 1985;7:539-52.

10. Davis JN. An experimental study of hiccup. Brain 1970;93:851-72. 